\title{
A comparative study of EUV absorber materials using lensless actinic imaging of EUV photomasks
}

\author{
S. Fernandez ${ }^{\text {a* }}$, D. Kazazis ${ }^{\mathrm{a}}$, R. Rajeev ${ }^{\mathrm{a}}$, I. Mochi ${ }^{\mathrm{a}}$, P. Helfenstein ${ }^{\mathrm{a}}$, S. Yoshitake ${ }^{\mathrm{b}}$ and Y. Ekinci ${ }^{\mathrm{a}}$ \\ ${ }^{a}$ Paul Scherrer Institute, Villigen PSI, Villigen, CH-5232, Switzerland \\ ${ }^{\mathrm{b}}$ NuFlare Technology, Inc., 8-1Shinsugita-cho, Isogo-ku, Yokohama 235-8522, Japan
}

\begin{abstract}
For EUV photomasks, high-k absorber materials represent a potential strategy to effectively mitigate mask 3D effects which are getting more prominent as the scanners' NA increases. The performance of RESCAN, our actinic lensless imaging microscope is evaluated through three different absorber materials (HSQ, TaBN, and Ni) together with the imaging properties of the materials themselves. Defect maps for each material are analyzed and compared.
\end{abstract}

Keywords: EUV lithography, EUV absorber, photomask, actinic inspection, coherent diffraction imaging

\section{INTRODUCTION}

Extreme ultraviolet (EUV) photomask technology, including fabrication, characterization, and inspection, is of key importance to make EUV lithography a cost-effective technology for high-volume semiconductor manufacturing. In this context, one of the core challenges is the mitigation of mask 3D effects such as pitch-dependent focus shift, induced pattern asymmetries and image contrast loss. Indeed, mask topography effects become more important as the mask feature sizes decrease, so that their origin and influence in aerial images must be carefully investigated and mitigated [1, 2].

Among the strategies to reduce the impact of mask 3D effects, reducing the thickness of the absorber layer, i.e., changing the material of the absorber stack with a high-k absorber while keeping the optical contrast similar, has been widely studied with numerical simulations [1-5]. Nevertheless, the fabrication of such samples remains challenging [3]. A direct experimental comparison between different absorbers and the impact of the chosen materials on aerial image formation is of crucial importance. By using actinic inspection, the amount or/and the kind of defects found within a mask may help to discard or promote a certain material. Furthermore, the use of different absorber materials allows the possibility of evaluating the resolution of the inspection tool itself as a function of the contrast for a given thickness of the chosen absorber stack.

In this paper, the imaging performance of the RESCAN (Reflective EUV Mask Scanning Lensless Imaging) tool on masks with three different absorber materials is presented. RESCAN is used for EUV mask inspection and it offers high resolution by employing coherent diffraction imaging (CDI) methods to retrieve both the magnitude and phase of the sample, thereby allowing the localization of both amplitude and phase defects with a very high sensitivity [6-8].

In previous works, we have demonstrated the capability of RESCAN to detect programmed defects as small as $50 \mathrm{~nm}$ on random patterns fabricated in-house[8]. In our studies, the absorber consisted of a hydrogen silsesquioxane (HSQ) layer with $140 \mathrm{~nm}$ thickness. Here, we use an analogous sample to be compared with a state-of-the-art EUV mask, which is a 70-nm-thick TaBN absorber, and a 60-nm-thick Ni layer, where the latter is among the preferred materials to optimize the absorber physical properties [3, 4, 9]. In Section 2, we explain the choice of these absorber materials and their optical properties and describe the samples investigated during the experiments presented here (Sec. 2.1). Then, the instrumental setup and technique employed in RESCAN are described (Sec. 2.2). The results in Section 3 show the comparative analysis of the imaging properties of different samples as well as the die-to-die inspection results.

*sara.fernandez@psi.ch

Extreme Ultraviolet (EUV) Lithography IX, edited by Kenneth A. Goldberg, Proc. of SPIE Vol. 10583, 105831H · C 2018 SPIE · CCC code: 0277-786X/18/\$18 · doi: 10.1117/12.2297381 


\section{EUV MASK ABSORBER MATERIALS AND INSPECTION EVALUATION}

EUV mask stacks consist of a MoSi multilayer, generally composed of 40 to 50 bi-layers, a Ru-based capping layer atop, and an absorber structure, as sketched in Fig. 1. The pattern is defined by selectively etching the absorber material on top of the multilayer (ML) mirror and the interaction of the incident light with the mirror itself. The contrast in the image is provided by the intensity difference between the light reflected by the mirror and the light transmitted through the absorber layer. Ta-based materials, e.g. TaN and TaBN, provide high absorption and are the state-of-the-art in EUV lithography $(\lambda=13.5 \mathrm{~nm})$. Nevertheless, a thickness of $70 \mathrm{~nm}$ is needed to ensure acceptable absorption [3], which induces the so-called mask $3 \mathrm{D}$ effects in the resulting projected images at non-zero angle of incidence. Moreover, these effects are likely to become more prominent as the pattern critical dimension decreases. Because the insertion of EUV lithography at $7 \mathrm{~nm}$ node is currently planned, different routes to mitigate these effects are being investigated, e.g., completely removing the absorber layer and etching the ML [10,11], changing the EUV mask structure [12], introducing phase shifting masks [13] or considering other absorber materials that have stronger absorption, i.e. higher k-values than $\mathrm{Ta}$, such as $\mathrm{Ni}$ or $\mathrm{Co}[1,4]$. Here, we focus on the choice of alternative materials and evaluate the imaging performances after the reduction of the absorber thickness.

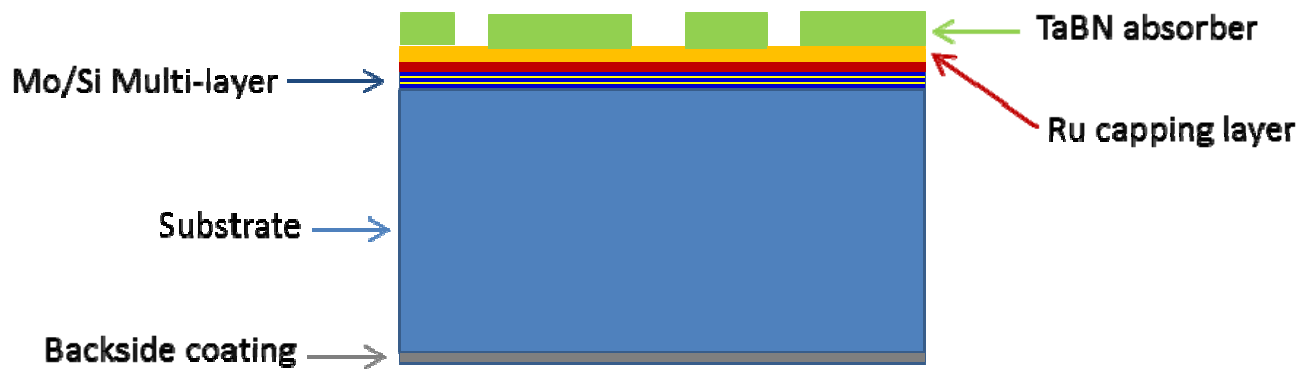

Figure 1. Schematic of the Ta-based EUV photomask measured in this work, which is the state-of-the-art in EUV photomask technology.

\subsection{Samples description}

When choosing the most suitable absorber material for EUV masks, several factors play a role, such as their optical properties at EUV wavelength, the feasibility of patterning with standard nanofabrication methods and the line-edge roughness of the resulting absorber pattern. The contrast between the absorbed and reflected light must be enhanced. As previously mentioned, the absorber thickness has to be reduced as much as possible to minimize proximity effects due to the oblique incident illumination. Hence, a high absorption coefficient $(k)$ and a refractive index $(n)$ as close to 1 as possible are desirable. The optical properties of the absorbers evaluated in this study are displayed in Table 1, in which we have included the thickness of the layers forming our samples. Note that, in the case of $\mathrm{Ni}$, the optimal thickness depends on the absorber coefficient and could be optimized [4,14]. However, obtaining thin, smooth and defect-free $\mathrm{Ni}$ layers on multilayer stacks without damaging the ML is extremely difficult $[15,16]$. Also, in the case of HSQ, the index of refraction depends on the development parameters after electron beam exposure, since density and composition may vary [17]. The given range corresponds to a density between $1.57 \mathrm{~g} / \mathrm{cm}^{3}$ and $2.17 \mathrm{~g} / \mathrm{cm}^{3}[18]$.

Table 1. Optical properties and thickness of the materials chosen as absorbers in this study $[6,15]$.

\begin{tabular}{|l|c|c|c|}
\hline & HSQ & TaBN & Ni \\
\hline Index of refraction $(n)$ & $0.9781-0.9843$ & 0.925 & 0.948 \\
\hline Absorber coefficient $(k)$ & $0.005-0.008$ & 0.044 & 0.073 \\
\hline Thickness $(n m)$ & 140 & 70 & 60 \\
\hline Attenuation length $(\mathrm{nm})$ & $217-135$ & 22.7 & 14.7 \\
\hline
\end{tabular}


In order to compare the influence of different absorber materials on the CDI reconstructions, we used a TaBN EUV mask (see Fig. 1) manufactured by Nuflare Technology Inc. and Dai Nippon Printing Co., Ltd., representing the state-of-theart absorber stack. We manufactured EUV masks with HSQ and Ni absorber layers in-house. Both layers were deposited on a silicon wafer with a MoSi multilayer designed for high reflectivity at $6^{\circ}$ angle of incidence and $13.5 \mathrm{~nm}$ wavelength. We chose HSQ due to its patterning simplicity. HSQ is a high-resolution, negative-tone e-beam resist, yielding $\mathrm{SiO}_{2}$ patterns subsequent to exposure and development. For the fabrication of the Ni sample, we carried out extensive process optimization to obtain high quality patterns despite the fact that $\mathrm{Ni}$ is prone to produce high line-edge roughness due to its crystallinity. We note that the present thicknesses of the HSQ and Ni absorbers are not optimized.

The layout of the EUV masks consists of a non-periodic pattern with horizontal power rails and extends over an area of $15 \times 16 \mu^{2}$, as shown in Fig. 2a). SEM images of the three samples are displayed in Figs. 2b)-d). The measured critical dimension (CD) was $114.4 \mathrm{~nm}$ for the TaBN sample, and $218 \mathrm{~nm}$ for the samples fabricated in-house (HSQ and Ni). Every sample contained a single programmed defect, consisting of an extrusion in the center of the pattern, as will be further explained in Sec. 3.2.

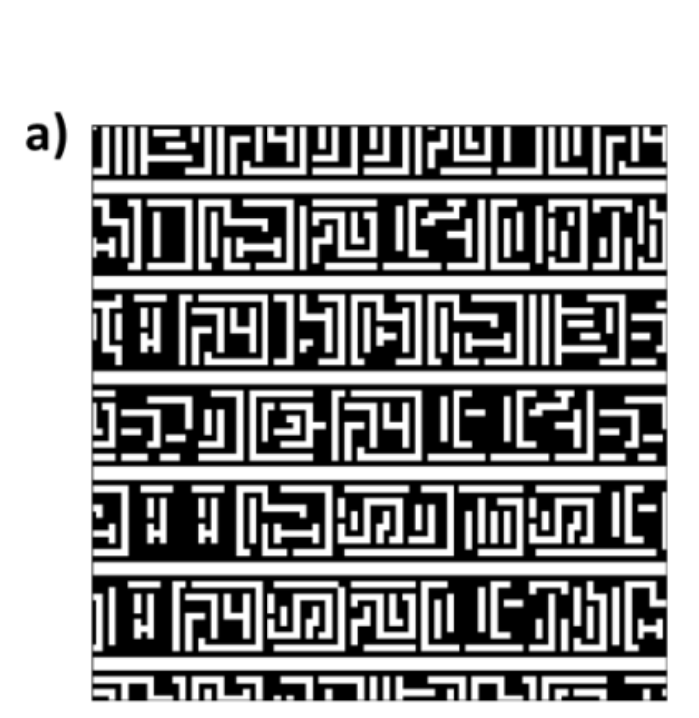

b)
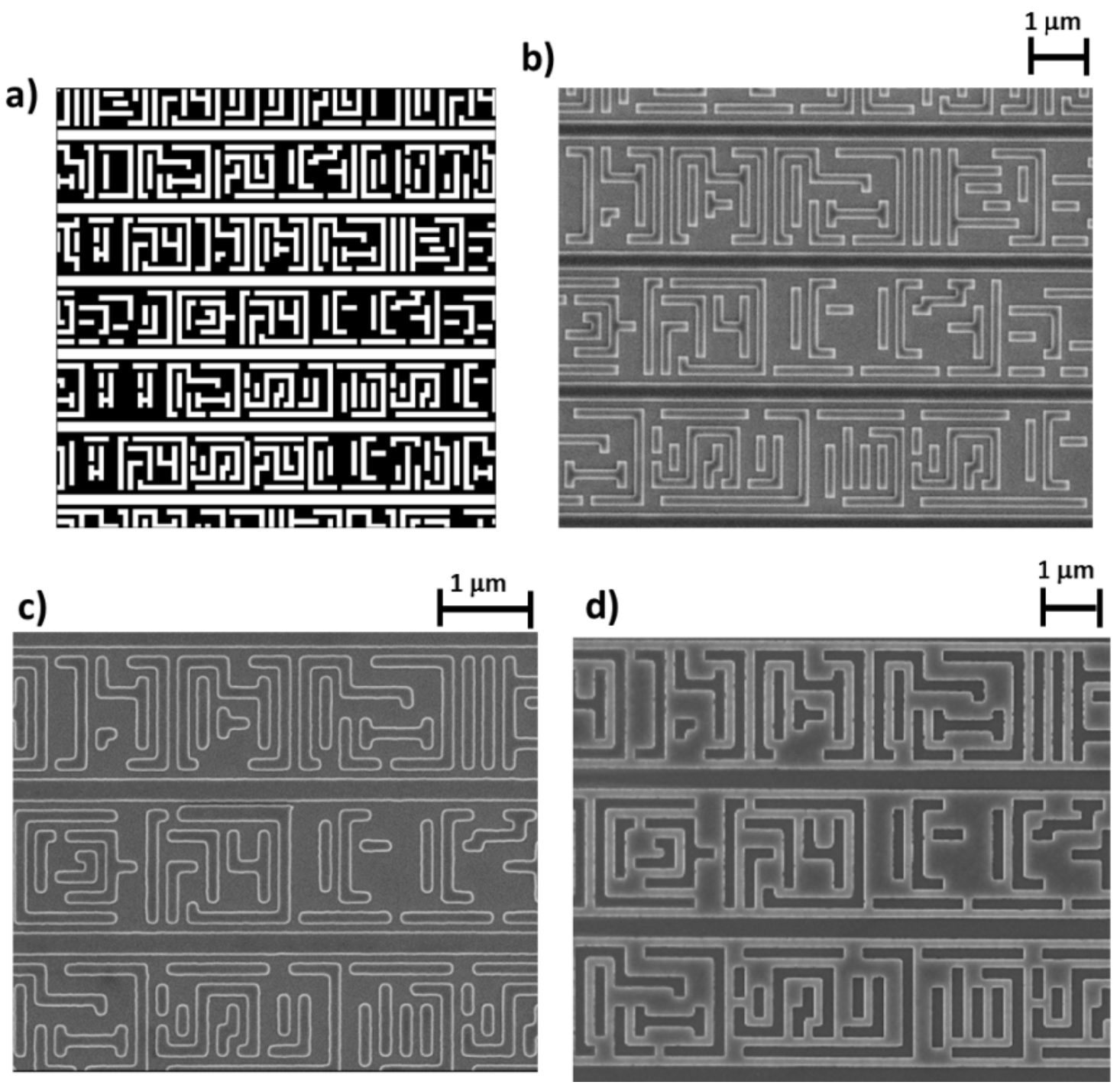

Figure 2. Layout of the unit cell of the random pattern $\left(15 \times 16 \mu \mathrm{m}^{2}\right)$ (a) and high resolution SEM images on the center of the fields of the HSQ (b), TaBN (c) and Ni (d) absorber samples, respectively. 


\subsection{Methods}

In order to investigate the masks described in Section 2.1., we employed a scanning coherent diffraction method known as ptychography. The three photomasks were inspected using the RESCAN tool installed at the XIL-II beamline at the Swiss Light Source (SLS) [19]. The incoming coherent beam $(\lambda=13.5 \mathrm{~nm})$ was focused using a condenser and a folding mirror with an incident angle of $6^{\circ}$, as depicted in Fig. 3. Detailed descriptions of the beamline and set-up can be found elsewhere [8], [19], [20].

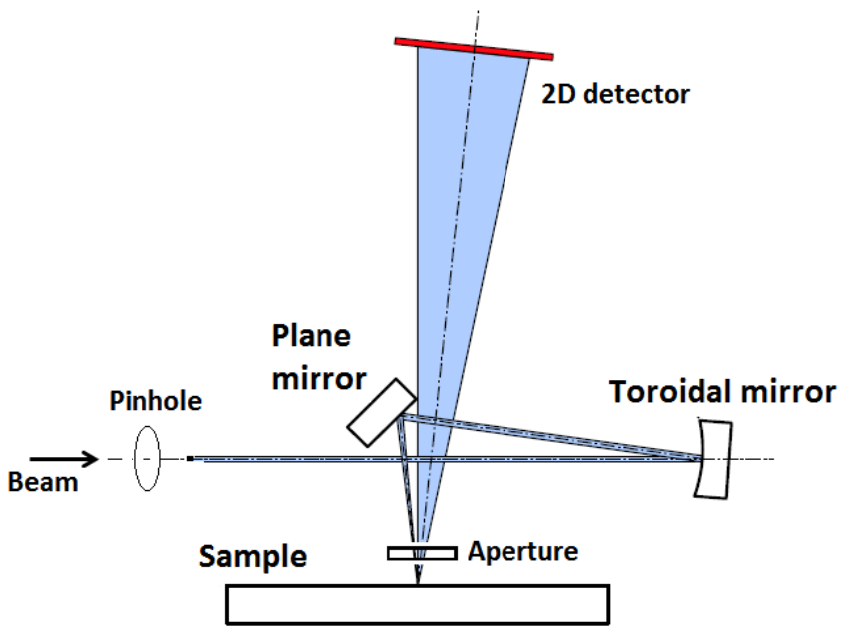

Figure 3. RESCAN experimental set-up at the XIL-II beamline. The aperture on top of the sample was used for the HSQ sample in order to avoid scattering from the blank ML outside the patterned field.

The masks were scanned with a high-precision piezoelectric positioning stage (accuracy of $\pm 3 \mathrm{~nm}$ ), while the scattered intensity from the reflective mask was collected by a two-dimensional detector with $2048 \times 2048$ pixels of $13.5 \mu \mathrm{m}$ size. Given the spot size (FWHM) of the incident illumination of about $3 \mu \mathrm{m}$ on the sample plane, a step size of $1 \mu \mathrm{m}$ was chosen to guarantee a minimum overlap of $60 \%$.

In order to record the higher spatial frequencies, longer exposures times are warranted. However, this leads to saturation at lower frequencies prevalent in the CCD camera. To overcome this, several diffraction patterns with different exposure times at every scan position were recorded, hence ensuring the coverage of both low and high spatial frequencies without saturation at a high signal-to-noise ratio. The diffraction data sets were then processed with the iterative algorithms [21, 22 ] to recover simultaneously both the amplitude and phase maps of the masks as well as the complex illumination probe field.

\section{EXPERIMENTAL RESULTS}

\subsection{CDI results: comparison between absorbers}

Figure 4 shows the reconstructed actinic images (amplitude maps) of about $10 \times 10 \mu \mathrm{m}^{2}$ area of the masks made by the HSQ and Ni absorbers and the equivalent one of about $6 \times 6 \mu \mathrm{m}^{2}$ of the TaBN absorber (smaller CD). The achieved pixel resolution is $34 \mathrm{~nm}$. As expected, the retrieved images show an enhancement of contrast in the case of the thinnest absorber, i.e., the $\mathrm{Ni}$ one. The reconstructed amplitude images reproduce in both cases the mask design with high fidelity. 

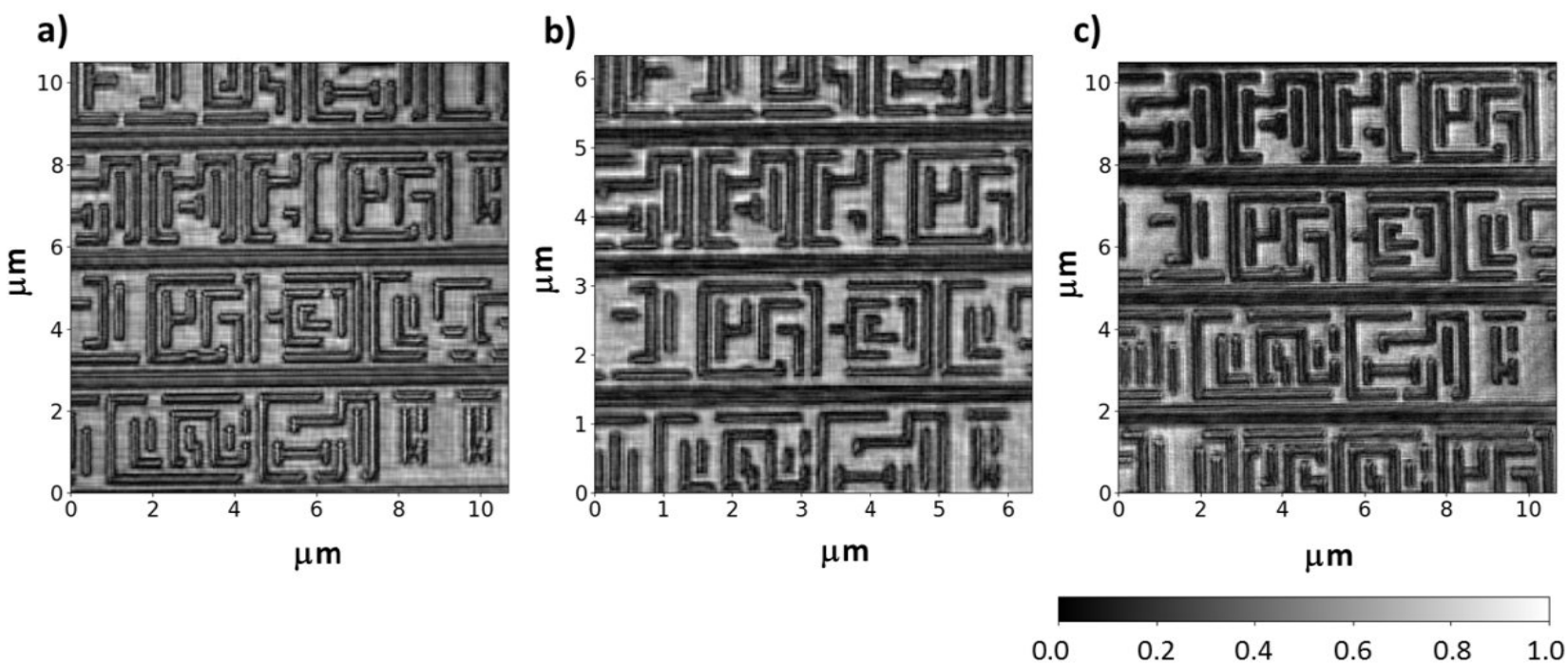

Figure 4. Zoomed image section of the reconstructed magnitudes of the a) HSQ, b) TaBN and c) Ni absorber masks. The color bar applies to all images.

The retrieved phase maps are shown in Fig. 5. The phase contrast measured from the reconstructed images yields phase differences of $\Delta \phi_{H S Q}=2.72 \pm 0.2 \mathrm{rad}, \Delta \phi_{T a B N}=2.19 \pm 0.3 \mathrm{rad}$ and $\Delta \phi_{N i}=2.74 \pm 0.5 \mathrm{rad}$, respectively. For absorber materials, the phase change is related to the thickness of the layer as follows [8]:

$$
\Delta \phi=\frac{4 \pi h}{\lambda}\left(\frac{1}{\cos \theta}-\frac{n^{2}}{\sqrt{n^{2}-\sin ^{2} \theta}}\right)
$$

where $h$ is the height of the sample, $\lambda$ the wavelength, and $\theta_{i}$ the incident angle. For the thickness and refractive index given in Table 1, we find that the measured phases are in good agreement with the theoretical ones, confirmed by atomic force microscopy, the latter being $\Delta \phi_{H S Q^{I}}=2.03 \mathrm{rad}, \Delta \phi_{H S Q^{I}}=3.04 \mathrm{rad}, \Delta \phi_{T a B N}=2.258 \mathrm{rad}$ and $\delta \phi_{N i}=2.89$. As expected for the given thicknesses, the phase contrast is highest in the case Ni.
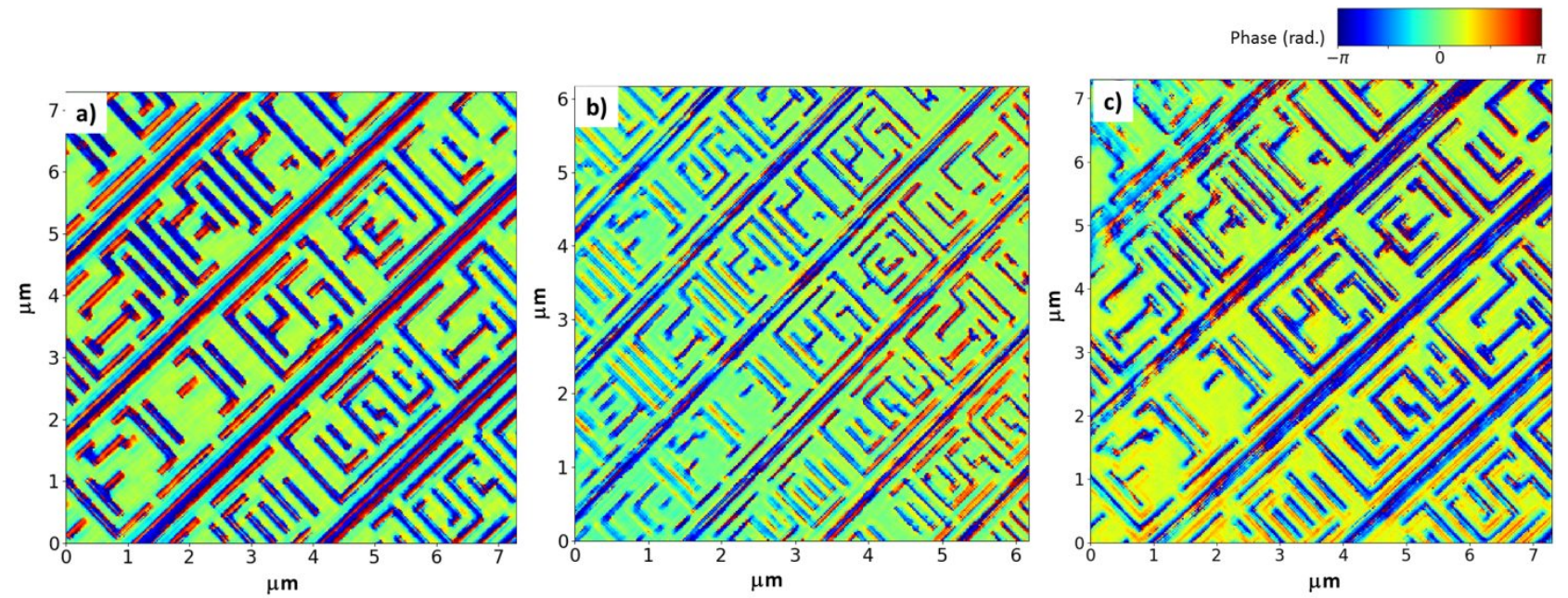

Figure 5. Zoomed image of the center of the reconstructed phase maps of the a) HSQ, b) TaBN and c) Ni absorber masks. The color bar applies to all maps. 
The present study, yielding high-contrast images, shows the capability of RESCAN to perform EUV actinic mask inspection with very good spatial resolution and sensitivity, independently of the chosen absorber material. Our study shows that a thin absorber improves the magnitude contrast, whereas HSQ gives a strong phase contrast with low amplitude contrast.

\subsection{Actinic inspection and defect sensitivity}

As introduced in Section 2.1, programmed defects were printed on every mask. Next to the field depicted in Sec. 2, Fig. 2a), a second pattern, equal to the previous one but containing a programmed defect, was reproduced. The defect consisted of an extrusion of $64 \times 32 \mathrm{~nm}^{2}$ in the TaBN mask, and $269 \times 70 \mathrm{~nm}^{2}$ size in the HSQ and Ni absorber masks due to the different CDs.

A die-to-die inspection can be performed based on the magnitude or phase maps using the reconstructed images. Zooms on the amplitude maps of the reference and defect fields are displayed in Fig. 6, first and second columns respectively. The extrusion is circled in red in the reconstructed amplitude. Every row corresponds to one material: HSQ, TaBN, and Ni from top to bottom.

On the third column of Fig. 6, the difference between the defect and the reference images is displayed for every absorber material. The difference was subtracted after alignment of the features to obtain the defect maps, in which the defect in the TaBN photomask turns out to be resolved best, despite its smaller size in both directions. For a better visualization of the intensity arising from the defect, a thresholded defect map is shown in the fourth column of the same Figure.

a)

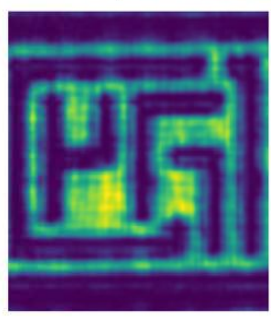

b)

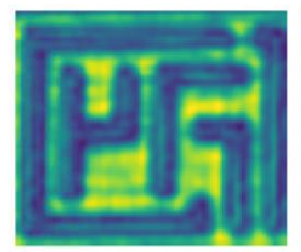

c)

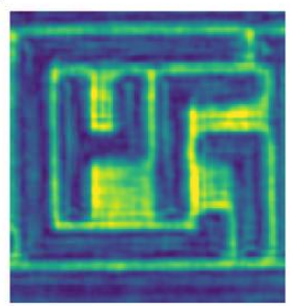

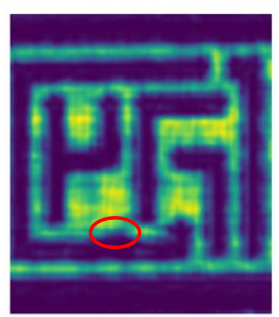
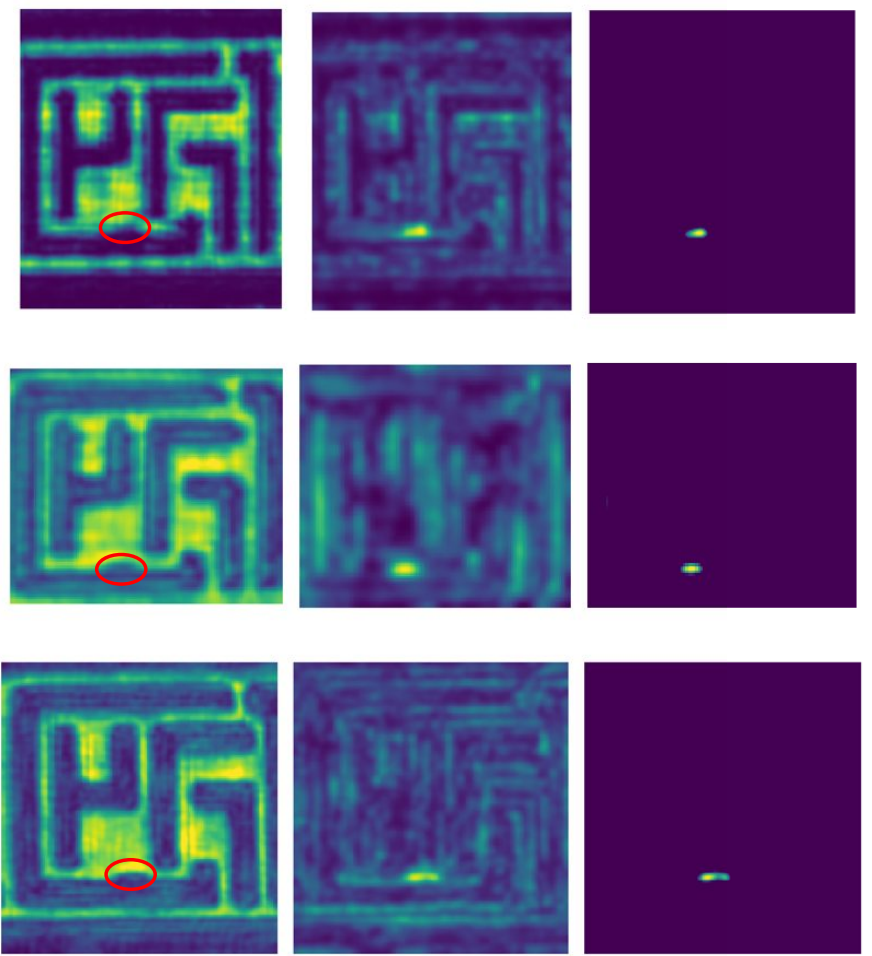

Figure 6. From left to right: reference and defect structures, defect maps and thresholded defect map of the samples fabricated with HSQ (a), TaBN (b) and $\mathrm{Ni}$ (c) as absorber materials. 
The defect signal-to-noise ratio (SNR) of these images can be calculated using the definition [8]:

$$
S N R=\frac{\overline{I_{D}}-\overline{I_{A}}}{\operatorname{std}(A)}
$$

where $\overline{I_{D}}$ and $\overline{I_{A}}$ are the average magnitude values calculated on the defect pixels of the image and on the whole image, respectively, while $\operatorname{std}(A)$ is the standard deviation of the magnitude of the image. The results are summarized in Table 2. The highest SNR was found for TaBN. We note that the reported SNR values are based on the average rather than on the integrated signal of all defect pixels and are therefore independent of the footprint of the defects.

Table 2. Defect sizes on the measured sample and signal-to-noise ratio (SNR).

\begin{tabular}{|l|c|c|c|}
\hline & HSQ & TaBN & Ni \\
\hline Defect size $\left(\mathrm{nm}^{2}\right)$ & $269 \times 70$ & $64 \times 35$ & $269 \times 70$ \\
\hline SNR & 6.85 & 7.89 & 7.64 \\
\hline Thickness $(\mathrm{nm})$ & 140 & 70 & 60 \\
\hline
\end{tabular}

\section{CONCLUSIONS AND OUTLOOK}

Three EUV photomasks with different absorber materials, i.e., HSQ, TaBN, and Ni, were prepared and evaluated from the actinic inspection point of view. Lensless imaging showed a better contrast for the thinnest layer, Ni, followed by TaBN and HSQ. On the other hand, the retrieved phases match the calculated ones from the height of the absorber stacks.

Programmed defects of sizes ranging from 35 to $70 \mathrm{~nm}$ minimum lateral sizes could be located and evaluated in RESCAN through a die-to-die approach. A higher SNR was found for the TaBN absorber despite the fact that it exhibited a lower contrast than Ni. Further investigations are necessary in order to generalize this result and make conclusions about the defect detection sensibility as a function of the material. A reduction of the Ni thickness would be advantageous for the comparison of the materials, as well as the reproduction of the same layout and CDs.

Manufacturability of EUV photomasks with alternative absorber materials remains nevertheless a concern and needs further work. In this paper we have shown that RESCAN is able to perform actinic inspection in any of the proposed materials, achieving high resolution and defect sensitivity and opening routes towards the study of other kinds of defects. Potentially, phase defects and shadowing effects at smaller pitches could be studied by taking advantage of the direct measurement of the phase.

\section{ACKNOWLEDGEMENTS}

The authors would like to thank Markus Kropf, Michaela Vockenhuber, and Li-Ting Tseng for their excellent support during the experiments and Shingo Yoshikawa, Dai Nippon Printing Co., Ltd, Japan, for providing the TaBN masks. Part of this work was performed at the Swiss Light Source (SLS), Paul Scherrer Institut, Switzerland.

\section{REFERENCES}

[1] P. Yan et al., "EUV mask absorber characterization and selection," Proc. SPIE 4066, 116-124 (2000).

[2] A. Erdmann, P. Evanschitzky, and T. Fühner, "Mask diffraction analysis and optimization for EUV masks," Proc. SPIE 7271, 72711E (2009). 
[3] A. Rastegar, M. House, R. Tian, T. Laursen, A. Antohe, and P. Kearney, "Study of alternative capping and absorber layers for extreme ultraviolet (EUV) masks for sub-16nm half-pitch nodes," Proc. SPIE 90480, 9048L (2014).

[4] V. Philipsen et al., "Reducing EUV mask 3D effects by alternative metal absorbers," Proc. SPIE 10143, 1014310 (2017).

[5] F. Scholze, C. Laubis, K. V. Luong, and V. Philipsen, "Update on optical material properties for alternative EUV mask absorber materials," Proc. SPIE 10446, 1044609 (2017).

[6] P. Helfenstein, I. Mohacsi, R. Rajeev, and Y. Ekinci, "Scanning coherent diffractive imaging methods for actinic extreme ultraviolet mask metrology," J. MicroNanolithography MEMS MOEMS 15(3) 034006 (2016).

[7] I. Mochi et al., "RESCAN: an actinic lensless microscope for defect inspection of EUV reticles," $J$. MicroNanolithography MEMS MOEMS 16(4), 041003, (2017).

[8] I. Mochi et al., "Actinic inspection of EUV reticles with arbitrary pattern design," Proc. SPIE 10450, 1045007 (2017).

[9] O. Wood et al., "Alternative materials for high numerical aperture extreme ultraviolet lithography mask stacks," Proc. SPIE 9422, 94220I (2015).

[10] B. L. Fontaine et al., "Architectural choices for EUV lithography masks: patterned absorbers and patterned reflectors," Proc. SPIE, 5374, 300-311 (2004).

[11] L. V. Look et al., "Alternative EUV mask technology to compensate for mask 3D effects," Proc. SPIE 9658, 96580I (2015).

[12] Y. Deng, B. M. L. Fontaine, H. J. Levinson, and A. R. Neureuther, "Rigorous EM simulation of the influence of the structure of mask patterns on EUVL imaging," Proc. SPIE 5037, 302-314 (2003).

[13] B. L. Fontaine et al., "Demonstration of phase-shift masks for extreme-ultraviolet lithography," Proc. SPIE 6151, 61510A (2006).

[14] T. Kamo, H. Aoyama, T. Tanaka, and O. Suga, "Effects of mask absorber thickness on printability in EUV lithography with high resolution resist," Proc. SPIE, 7028, 70281R (2008).

[15] D. Hay et al., "Thin absorber extreme ultraviolet photomask based on Ni-TaN nanocomposite material," Opt. Lett. 41(16), 3791-3794 (2016).

[16] C. M. Gonzalez et al., "Evaluation of Mask Repair Strategies Via Focused Electron, Helium and Neon Beam Induced Processing for EUV Applications," in Proc. SPIE 9048, 90480M (2014).

[17] B. L. Henke, E. M. Gullikson, and J. C. Davis, "X-Ray Interactions: Photoabsorption, Scattering, Transmission, and Reflection at E = 50-30,000 eV, Z =1-92," At. Data Nucl. Data Tables, 54(2), 181342 (1993).

[18] H.-J. Lee, E. Lin, H. Wang, W.-L. Wu, W. Chen, and E. Moyer, "Structural Comparison of Hydrogen Silsesquioxane Based Porous Low-k Thin Films Prepared with Varying Process Conditions," Chem. Mater.14, 1845-1852 (2002).

[19] R. Rajendran et al., "Towards a stand-alone high-throughput EUV actinic photomask inspection tool: RESCAN," Proc. SPIE 10145, 101450N (2017).

[20] N. Mojarad, J. Gobrecht, and Y. Ekinci, "Interference lithography at EUV and soft X-ray wavelengths: Principles, methods, and applications," Microelectron. Eng. 143, 55-63 (2015).

[21] P. Thibault, M. Dierolf, O. Bunk, A. Menzel, and F. Pfeiffer, "Probe retrieval in ptychographic coherent diffractive imaging," Ultramicroscopy, 109(4), 338-343 (2009).

[22] A. M. Maiden and J. M. Rodenburg, "An improved ptychographical phase retrieval algorithm for diffractive imaging," Ultramicroscopy 109(10), 1256-1262 (2009). 